\title{
Nonlinear Stability of an Undercompressive Shock for Complex Burgers Equation
}

\author{
Tai-Ping Liu', Kevin Zumbrun² \\ 1 Stanford University, Department of Mathematics, Standford, CA 94305-2060, USA \\ 2 Indiana University, Department of Mathematics, Bloomington, IN 47405, USA
}

Received: 1 March 1994

\begin{abstract}
Though there is strong numerical evidence for the stability of undercompressive shocks, their stability has not been verified analytically. In particular, the energy methods used to analyze stability of standard shocks do not apply. Here, we present the first proof of stability for a particular undercompresive shock, the real Burgers shock considered as a solution of the complex Burgers equation. Our analysis is by direct calculation of the Green's function for the linearized equations, combined with pointwise estimates of nonlinear effects. A benefit of this method is to obtain fairly detailed information about the solution, including $L^{1}$ behavior, and rates of decay in different regions of space.
\end{abstract}

\section{Introduction}

Undercompressive shock wave solutions of $n \times n$ systems of conservation laws are viscous profiles with less than the $n+1$ entering characteristics of a standard (Lax) shock, [Lax]. They can arise in association with a loss of strict hyperbolicity, as can overcompressive shocks, which are viscous profiles with more than $n+1$ entering characteristics.

The appearance of these nonclassical waves makes the solution of Riemann problems considerably more complicated. For example, overcompressive shocks are generally associated with loss of uniqueness $[\mathrm{F}]$, while undercompressive waves depend sensitively on the way viscosity is introduced via parabolic regularization [I-Ma-Pl]. Both types of wave violate the Lax characteristic condition for linearized stability. It has been demonstrated for the case of overcompressive shocks in [L, 3, F-L, L-Xi] that this confusion at the hyperbolic level can be resolved by closer study of an encompassing parabolic regularization. In particular, there has evolved [L3] a general principle for admissibility of waves, which might be called the "stable viscous profile" condition. That is, an admissible shock must not only have a viscous profile, but that profile must have sufficient stability properties to be persistent as a parabolic solution, even as viscosity goes to zero.

These issues motivate the study of the stability and behavior of undercompressive profiles as solutions of parabolic conservation laws. In [Zu-Pl-Ma], a heuristic 
model was developed for the behavior of undercompressive profiles under perturbation, and tested numerically. In all runs, undercompressive shocks showed extremely robust stabillity, supporting their admissibility as hyperbolic waves. Further, it can be verified analytically [Che-Xi, Zu.2] that they are stable as hyperbolic solutions, by a Glimm scheme argument, provided that the viscous profile condition is adopted as an admissibility requirement, with the form of viscosity specified. This can be regarded as a consistency result.

However, to this date, there has been no proof of nonlinear parabolic stability for any undercompressive shock. The main difficulty is that standard methods for analyzing stability of shocks [Mat-Ni, G.1, L.1, Sz-Xi] require a priori knowledge of the asymptotic state of a perturbed shock. This is accomplished, following [L.1], by the ansatz that the asymptotic state consists of a translate of the original shock, plus diffusion waves in the modes with outgoing characteristics. These diffusion waves are essentially nonlinear heat kernels, carrying finite mass but the decaying in $L^{\infty}$. If we denote the unperturbed shock by $\Phi(x)$, the perturbation by $\bar{U}$, the translation by $d$, and the masses of the diffusion waves by $\rho_{i}$, then conservation of mass gives the relation

$$
\int_{-\infty}^{+\infty} \bar{U} d x=\int_{-\infty}^{+\infty}[\Phi(x-d)-\Phi(x)]+\sum \rho_{i} r_{i}
$$

where $r_{i}$ denotes the mode of propagation associated with the $i^{\text {th }}$ diffusion wave.

For a standard (Lax) shock, then $n-1$ outgoing masses $\rho_{i}$ and the translation $d$ are determined by the $n$ equations (1.1). However, an undercompressive shock has $n$ or more outgoing characteristics, and its asyptotic state is not determined.

Here, we study an illustrative example, for which the shock location, timeasymptotically, can be determined apriori, but the diffusion waves cannot. The complex Burgers equation,

$$
\begin{aligned}
\tilde{u}_{t}+1 / 2\left(\tilde{v}^{2}-\tilde{u}^{2}\right)_{x} & =\tilde{u}_{x x}, \\
\tilde{v}_{t}+(\tilde{u} \tilde{v})_{x} & =\tilde{v}_{x x}
\end{aligned}
$$

is a standard example of a type II quadratic flux model, in the classification scheme of [Sch-Sh]. We consider the stability under perturbation of the solution given by the real Burgers shock,

$$
\Phi(x)=\left(\begin{array}{c}
\varphi(x) \\
0
\end{array}\right)=\left(\begin{array}{c}
\tanh (x / 2) \\
0
\end{array}\right) .
$$

The Jacobian of the flux function of (1.2) is

$$
\left(\begin{array}{cc}
-\tilde{u} & \tilde{v} \\
\tilde{v} & \tilde{u}
\end{array}\right)
$$

Thus, along $\Phi(x)$, the modes of propagation, given by the right eigenvectors, are $r_{1}=\left(\begin{array}{l}1 \\ 0\end{array}\right)$ and $r_{2}=\left(\begin{array}{l}0 \\ 1\end{array}\right)$, and the associated characteristic speeds are $\lambda_{1}=-\varphi(x)$ and $\lambda_{2}=\varphi(x)$. Since the shock speed is zero, at $x=\mp \infty$, there is one entering and one leaving characteristic, so that $\Phi(x)$ is indeed undercompressive.

However, note that both outgoing characteristic modes are in the direction $r_{2}=$ $\left(\begin{array}{l}0 \\ 1\end{array}\right)$. Thus, we can combine $\rho_{-\infty} r_{-\infty}+\rho_{+\infty} r+_{\infty}$ into $\left(\rho_{-\infty}+\rho_{+\infty}\right) r_{2}$ and solve 
for the translation $d$ and the total outgoing mass $\left(\rho_{-\infty}+\rho_{+\infty}\right)$ by conservation of mass, in (1.1). By determining the correct translation in the asymptotic state, we can at least avoid the order one error resulting from the wrong shock location.

On the other hand, the possible error in the mass of diffusion waves still presents a problem in the standard energy method. Morever, the fact that the secondary characteristic speed $\lambda_{2}$ vanishes at $x=0$ is equally serious, as it prevents the "vertical estimate" [G.1] or weighting method [G.2] used to deal with the lack of compressivity in secondary fields. Thus, our example still retains many of the technical difficulties of the general case.

A nice feature of the complex Burgers equation is that, like the real Burgers equation, it has a great deal of helpful structure, making certain estimates possible by direct calculation. For example, an exact solution of the linearized equations around the shock profile can be determined.

The model is immediately illuminating at a naive level. The mode of propagation of the shock is clearly $r_{1}=\left(\begin{array}{l}1 \\ 0\end{array}\right)$, since it it actually a scalar Burgers shock along the $\tilde{u}$ axis, and the associated characteristic speed is $\lambda_{1}$. We can see that the classical division into fast and slow waves is not applicable here, since the characteristic speeds $\lambda_{1}$ and $\lambda_{2}$ cross as we traverse $\Phi(x)$ is neither fast nor slow, but rather "transitional" between fast and slow waves (cf. [I-Ma-Pl]). This situation is typical for undercompressive waves.

From this perspective, the label "undercompressive" is something of a misnomer. For, the primary wave field $\lambda_{1}$, associated with the mode of propagation of the shock, is equally compressive for either the standard (Lax) shock or an undercompressive shock, while the secondary fields are compressive for neither! We can already see that the mechanism for stability is also similar in either case. Perturbations in the primary, compresssive mode are carried in towards the shock, to cancel rapidly by diffusion, while perturbations is secondary modes are carried out towards infinity, to decay algebraically by diffusion in the absence of interaction, like heat kernels.

The difference is that in the standard case, the secondary waves always travel with speed different from the shock. In the undercompressive case, the speed of a secondary wave can coincide with the speed of the shock, leaving open the possibility of some kind of resonance in the solution. It is precisely this that we must show does not occur.

At an intuitive level, we can resolve this difficulty easily. When dissipation is taken into account, characteristics no longer propagate the solution. For a linear, parabolic system, the propagator of the solution is the Green's function. From this point of view, the proper generalization of the iterated characteristic methods used to study nonlinear hyperbolic problems [Co-Fr, Gl, L.2] is the iteration via Duhamel's principle, where a nonlinear source is propagated by the Green's function of the linearized problem.

When we explicitly calculate the Green's function for the linearized equations around the Burgers shock, we find that, in the secondary family, it consists simply of two waves, moving with speeds plus and minus one. Thus, even though the characteristic speed may vanish, the actual speeds at which secondary waves are propagated are always different from the shock speed, and resonance does not occur. However, to translate this simple observation into bounds on our iterated solution requires some rather careful estimates.

It may already be apparent from this description that it will be important in our analysis to track the location of different waves in the solution. We decompose the 
solution into waves of three main types, namely, the wedge

$$
W_{c} \triangleq \begin{cases}1 & -t \leqq x \leqq t \\ e^{\frac{-(x-t)^{2}}{4 C t}} & x \geqq t \\ e^{\frac{-(x+t)^{2}}{4 C t}} & x \leqq-t\end{cases}
$$

the moving heat kernels

$$
K_{C}^{ \pm}(x, t) \triangleq K\left(\frac{x \mp t}{C}, t\right)=\frac{1}{\sqrt{r \pi C t}} e^{-\frac{(x \mp t)^{2}}{4 C t}}
$$

and the stationary wave $e^{-|x| / 2 C}$, where the constant $C>1$ is to be determined later.

We will establish the following two theorems.

Theorem 1 Main Theorem. Let $C>1$ and $t_{0}$ be fixed, and let

$$
\left\{\begin{array}{c}
\tilde{u}_{0}(x) \\
\tilde{v}_{0}(x)
\end{array}\right\}=\Phi(x)+\left\{\begin{array}{l}
u_{0}(x) \\
v_{0}(x)
\end{array}\right\},
$$

with $\left|u_{0}(x)\right|,\left|v_{0}(x)\right| \leqq \delta e^{-x^{2} / 4 t_{0}}$ and

$$
\int_{-\infty}^{\infty} u_{0}(x) d x=0
$$

Then, for $\delta$ sufficiently small, (1.1) has a global solution

$$
\left\{\begin{array}{c}
\tilde{u}(x, t) \\
\tilde{v}(x, t)
\end{array}\right\}=\Phi(x)+\left\{\begin{array}{l}
u(x, t) \\
v(x, t)
\end{array}\right\}
$$

satisfying

$$
\begin{aligned}
u(x, t)= & O(1) \delta\left[\left(t+t_{0}\right)^{-3 / 2} W_{C}\left(x, t+t_{0}\right)+K_{C}^{-}\left(x, t+t_{0}\right)^{2}\right. \\
& \left.+K_{C}^{+}\left(x, t+t_{0}\right)^{2}+\left(t+t_{0}\right)^{-1 / 2} e^{-|x| / 2 C}\right] \\
v(x, t)= & O(1) \delta\left[K_{C}^{-}\left(x, t+t_{0}\right)+K_{C}^{+}\left(x, t+t_{0}\right)\right]
\end{aligned}
$$

where $O(1)$ is a constant depending only on $C, t_{0}$.

Theorem 2 ( $L^{1}$ Behavior). The perturbation $u$ converges to 0 in $L^{1}$ at the rate $t^{-1 / 2}$, while $v$ converges at rate $t^{-1 / 4}$ to a pair of heat kernels moving with speeds plus and minus 1 , respectively.

Remark. In $L^{p}$, the rates of convergence given in Theorem 2 become $t^{-1 / 2}$ for $u$ and $t^{-3 / 4+1 / 2 p}$ for $v$, i.e. $\left(\begin{array}{c}u \\ v\end{array}\right)$ converges to its asymptotic state as

$$
\left\{\begin{array}{ll}
t^{-1 / 2} & p \geqq 2 \\
t^{-3 / 4+1 / 2 p} & p \leqq 2
\end{array} .\right.
$$

These rates appear to be sharp. 
Plan of the paper. In Sect. 2, we examine the linearized equation around the shock profile, and determine a convenient formula for its Green's function. In Sect. 3, we use this formula and variation of constants to estimate the effects of different source terms in the linearized equations. This is the main section of the paper.

In Sect. 4, we divide the perturbation equations into a linear part and a nonlinear source term, and set up the standard iteration scheme for the solution. Using the estimates from Sect. 3, we show that certain pointwise bounds on the solutions are preserved in the iteration scheme. We thus obtain an a priori estimate which at once gives both global existence and decay of the perturbation.

In Sect. 5 , we slightly improve the basic ( $L^{1}$ bounded) stability result of Sect. 4 to obtain Theorem 1, and, after a further calculation, Theorem 2. Both calculations involve tricks separate from the rest of the paper. Finally, in Sect. 6, we state without proof the straightforward generalization of Theorem 1 to the higher derivatives of the solution.

We remark that the method of analysis introduced here gives detailed information about the decay of perturbations that has been obtained only recently [L.4] for standard (Lax) shocks. Previous gross estimates from semigroup theory, energy estimates, etc. were not sufficient even to show stability. This approach seems to be a useful combination of hyperbolic thinking, in particular in the resolution of the solution into distinct component waves, and standard parabolic techniques.

\section{The Linearized Equations}

Linearizing equations (1.2) around the shock profile $\Phi$ gives the decoupled system

$$
\begin{aligned}
& L_{1} u=u_{t}-(\phi(x) u)_{x}-u_{x x}=0, \\
& L_{2} u=v_{t}-(\phi(x) v)_{x}-v_{x x}=0,
\end{aligned}
$$

In this section, we derive integral representations for $L_{1}^{-1}$ and $L_{2}^{-1}$ by explicitly finding Green's functions for (2.1) (precisely speaking, for the integral of (2.1)) and (2.2)

Proposition 2.1. Let

$$
\begin{aligned}
& L_{1} u=\left(S_{1}\right)_{x}, \\
& L_{2} v=\left(S_{2}\right)_{x} .
\end{aligned}
$$

Then,

$$
\begin{aligned}
u(x, t)= & \int_{-\infty}^{\infty} u(y, 0) G_{1}(x, t ; y) d y \\
& +\int_{0}^{t} \int_{-\infty}^{\infty} G_{1}(x, t=s ; y) S_{1}(y, s) d y d s
\end{aligned}
$$

and

$$
\begin{aligned}
v(x, t)= & \int_{-\infty}^{\infty} v(y, 0) G_{2}(x, t ; y) d y \\
& +\int_{0}^{t} \int_{-\infty}^{\infty} G_{2}(x, t-s ; y) S_{2_{x}}(y, t) d y d s
\end{aligned}
$$


where

$$
\begin{gathered}
U(x, 0) \triangleq \int_{-\infty}^{x} u(y, 0) d y, \\
G_{1}(x, t ; y)=\left[\alpha^{-}(x) K_{x}^{+}(x-y, t)+\alpha^{+}(x) K_{x}^{-}(x-y, t)\right] \\
\left.+p-\alpha^{0}(x) K^{+}(x-y, t)+\alpha^{0}(x) K^{-}(x-y, t)\right], \\
G_{2}(x, t ; y)=\alpha^{-}(y) K^{-}(x-y, t)+\alpha^{+}(y) K^{+}(x-y, t),
\end{gathered}
$$

and

$$
\begin{gathered}
\alpha^{\mp}(x) \triangleq \frac{e^{\mp x / 2}}{e^{x / 2}+e^{-x / 2}} ; \quad \alpha^{0}(x) \triangleq(x)=-\alpha^{-^{\prime}}(x)=\frac{1}{\left(e^{x / 2}+e^{-x / 2}\right)^{2}}, \\
K^{ \pm}(x, t) \triangleq K(x \mp t, t)=\frac{1}{\sqrt{4 \pi t}} e^{\frac{-(x \mp t)^{2}}{4 t}}
\end{gathered}
$$

Proof. Equation (2.1) is just the linearized Burgers equation. From the first conservation law (1.8), it is natural to integrate this equation to obtain

$$
\mathscr{L}_{1} U=U_{t}-2 \phi(x) U_{x}-U_{x x}=0,
$$

where

$$
U(x, t) \triangleq \int_{-\infty}^{x} u(y, t) d t .
$$

Both Eqs. (2.1)' and (2.2) can be solved exactly by the method of weights introduced in [Sat]. Precisely, for

$$
\Lambda\left(x_{1}\right) \triangleq e^{\int_{0}^{x} 1 / 2 \phi(y) d y},
$$

the weighted variables $U \Lambda(x)$ and $u \Lambda^{-1}(x)$ both satisfy the dampled heat equation

$$
M w=w_{t}-w-w_{x x}=0 .
$$

The Green's function for $(2.11)$ is

$$
J(x, t ; y)=e^{-t} K(x-y, t),
$$

where $K(x, t)=\frac{1}{\sqrt{4 \pi t}} e^{-\frac{x^{2}}{4 t}}$ is the standard heat kernel. Thus, the Green's functions for $\left(2.1^{\prime}\right)$ and $(2.2)$ are $\mathscr{G}_{1}(s, t ; y)=\frac{\Lambda(y)}{\Lambda(x)} J(x, t ; y)$ and $G_{2}(x, t ; y)=\frac{\Lambda(x)}{\Lambda(y)} J(x, t ; y)$, respectively.

Evaluating

$$
\begin{aligned}
\Lambda(x) & =e^{\int_{0}^{x} 1 / 2 \tanh \left(\frac{y}{2}\right) d y} \\
& =e^{\log \cosh (x / 2)=\cosh \left(\frac{x}{2}\right)} \\
& =\cosh \left(\frac{x}{2}\right),
\end{aligned}
$$

and rearranging, we find (cf. [Zu.2]) that

$$
\mathscr{G}_{1}(x, t ; y)=\alpha^{-}(x) K^{+}(x-y, t)+\alpha^{+}(x) K^{-}(x-y, t),
$$


and

$$
G_{2}(x, t ; y)=\alpha^{-}(y) K^{-}(x-y, t)+\alpha^{+}(y) K^{+}(x-y, t),
$$

as in $(2.9-10)$. Note that the relation $G_{2}(x, t ; y)=\mathscr{G}_{1}(y, t ; x)$ follows from the fact that $\left(2.1^{\prime}\right)$ and (2.2) are adjoint.

Equation (2.5) now follows immediately, from (2.12) and Duhamel's principle.

Likewise, the integrated equation

$$
\mathscr{L}_{1} U=S_{1}
$$

has solution

$$
\begin{aligned}
U(x, t)= & \int_{-\infty}^{\infty} U(y, 0) \mathscr{G}_{1}(x, t ; y) d y \\
& +\int_{0}^{t} \int_{\infty}^{\infty} S_{1}(y S) \mathscr{G}_{1}(x, t-s ; y) d y d s,
\end{aligned}
$$

with $\mathscr{G}_{1}$ given in (2.11). Equation (2.4) follows upon differentiating in $x$, with the observation that

$$
\left(\mathscr{G}_{1}\right)_{x}=G_{1} \text {. }
$$

\section{Main Estimates}

In this section, we carry out the main analysis, using the representation derived in Proposition 2.2 to estimate the effects of different source terms on the solutions of the linearized equations.

Sources which are naturally associated with the modes of propagation of the linearized equations are the wedge

$$
W_{C} \triangleq \begin{cases}1 & -t \leqq x \leqq t \\ e^{\frac{-(x-t)^{2}}{4 C t}} & x \geqq t \\ e^{\frac{-(x+t)^{2}}{4 C t}} & x \leqq-t\end{cases}
$$

the moving heat kernels

$$
K_{C}^{ \pm}(x, t) \triangleq K\left(\frac{x \mp t}{C}, t\right)=\frac{1}{\sqrt{4 \pi C t}} e^{-\frac{(x \mp t)^{2}}{4 C t}},
$$

and the stationary wave $e^{-|x| / 2 C}$, which is asymptotic to $\alpha^{0}(x / 2 C)$. The constant $C>1$ is to be determined later.

Our aim is to prove the following two propositions:

Proposition 3.1. Let $L_{1} u=\left(S_{1}\right)_{x}, u(x, 0) \equiv 0$, and $C>1$.

(i) If

$$
\begin{aligned}
|S| & =O(1)\left[(t+1)^{-r} W_{C}(x, t+1)+(t+1)^{q} e^{-|x| / 2 C}\right], \quad \text { and } \\
\left|S_{x}\right| & =O(1) t^{-1 / 2}\left[(t+1)^{-r+1 / 2} W_{C}(x, t+1)+(t+1)^{-q+1 / 2} e^{-|x| / 2 C}\right],
\end{aligned}
$$

then

$$
|u|,\left|u_{x}\right|+O(1)\left[(t+1)^{-r+1 / 2} W_{C}(x, t+1)+(t+1)^{-\min \{q, r-1\}} e^{-|x| / 2 C}\right] .
$$


(ii) If

$$
\begin{aligned}
|S| & =O(1)(t+1)^{-p}\left[K_{C}^{-}(x, t+1)^{2}+K_{C}^{+}(x, t+1)^{2}\right] \quad \text { and } \\
\left|S_{x}\right| & =O(1) t^{-1 / 2}(t+1)^{-p}\left[K_{C}^{-}(x, t+1)^{2}+K_{C}^{+}(x, t+1)^{2}\right]
\end{aligned}
$$

then

$$
|u|,\left|u_{x}\right|+O(1)\left[(t+1)^{-p-1} W_{C}(x, t+1)+(t+1)^{\{-p-1 / 2\}} e^{-|x| / 2 C}\right] .
$$

Proposition 3.2. Let $L_{2} v=\left(S_{2}\right)_{x}, v(x, 0) \equiv 0$, and $C>1$. If

$$
\begin{gathered}
|S|=O(1)\left[(t+1)^{1 / 2} e^{-|x| / 2 C}+(t+1)^{-1}\right]\left[K_{C}^{-}(x, t+1)+K_{C}^{+}(x, t+1)\right], \\
\text { and }\left|S_{x}\right|=O(1) t^{-1 / 2}\left[K_{C}^{-}(x, t+1)+K_{C}^{+}(x, t+1)\right],
\end{gathered}
$$

or

(ii)

$$
\begin{aligned}
& |S|=O(1)\left[(t+1)^{1 / 2}\left[K_{C}^{-}(x, t+1)+K_{C}^{+}(x, t+1)\right],\right. \\
& \text { and }\left|S_{x}\right|=O(1) t^{-1}\left[K_{C}^{-}(x, t+1)+K_{C}^{+}(x, t+1)\right],
\end{aligned}
$$

then

$$
\begin{aligned}
|v| & =O(1)\left[K_{C}^{-}(x, t+1)+K_{C}^{+}(x, t+1) \quad\right. \text { and } \\
\left|v_{x}\right| & =O(1)(t+1)^{-1 / 2}\left[K_{C}^{-}(x, t+1)+K_{C}^{+}(x, t+1)\right] .
\end{aligned}
$$

To establish Proposition 3.1, we first prove two partial results.

Lemma 3.1.a. If

$$
|S|=O(1)(t+1)^{-r} W_{C}(x, t+1),
$$

then

$$
|u|=O(1)\left[(t+1)^{-r+1 / 2} W_{C}(x, t+1)+(t+1)^{-r+1} e^{-|x| / 2 C}\right] .
$$

Proof. Using the relations $K^{ \pm}(y, s)=O(1) s^{1 / 2} K_{\varepsilon}^{ \pm}(y, s) K_{C}^{ \pm}(y, s), \quad(2.4)$, and $K^{ \pm} x(y, s)=O(1) K_{\varepsilon}^{ \pm}(y, s) K_{C}^{ \pm}(y, s)$, we have

$$
|u(x, t)|=O(1) \int_{0}^{t} \int_{-\infty}^{\infty}\left(I_{1}^{+}+I_{2}^{+}+I_{2}^{-}+I_{2}^{-}\right) d y d s
$$

where

$$
\begin{aligned}
I_{1}^{ \pm}= & \alpha^{\mp}(x) K_{x}^{ \pm}(x-y, t-s) S(y, s) \\
= & O(1)(s+1)^{-r} \alpha^{\mp}(x) K_{C}^{ \pm}(x-y, t-s) \\
& \times K_{\varepsilon}^{ \pm}(x-y, t-s) W_{C}(y, s+1), \\
I_{2}^{ \pm}= & \mp \alpha^{0}(x) K^{ \pm}(x-y, t-s) S(y, s) \\
= & O(1)(t-s)^{1 / 2}(s+1)^{-r} \alpha^{0}(x) K_{C}^{ \pm}(x-y, t-s) \\
& \times K_{\varepsilon}^{ \pm}(x-y, t-s) W_{C}(y, s+1) .
\end{aligned}
$$


We rely on the identity

$$
\sup _{y} e^{-\frac{(x-y)^{2}}{4 C(t-s)}} e^{-\frac{y^{2}}{4 C s}}=e^{-\frac{x^{2}}{4 C t}}
$$

Applying the change of coordinates $x^{\prime}=x \mp t, y^{\prime}=y-\sigma$ and letting $\sigma$ vary between $-(s+1)$ and $(s+1)$, we find that

$$
\begin{aligned}
& \sup _{y} K_{C}^{ \pm}(x-y, t-s) W_{C}(y, s+1) \\
& \quad=\frac{1}{\sqrt{4 \pi C(t-s)}} \min \left\{W_{C}(x, t+1) W_{C}(x \mp 2(t-s), t+1)\right\} .
\end{aligned}
$$

Note that for $s \leqq t / 8$,

$$
\begin{gathered}
W_{C}(x \mp 2(t-s), t+1) \leqq \begin{cases}1 & x \gtrless \pm 3 t / 4 \mp 1 \\
e^{-\frac{(x \mp 3 t / 4 \mp 1) 2}{4 C t}} & x \geqq \pm 3 t / 4 \mp 1,\end{cases} \\
a^{\mp}(x) \leqq \begin{cases}1 & x \gtrless 0 \\
e^{-|x|} & x \gtrless 0,\end{cases} \\
\left|\alpha^{0}(x)\right| \leqq e^{-|x|} .
\end{gathered}
$$

Thus, for $s \leqq t / 8, C \geqq 1$,

$$
\alpha^{\mp}(x) W_{C}(x \mp 2(t-s), t+1)=O(1) e^{-|x| / 2 C} e^{-b t},
$$

for some positive constant $b$. Using (3.18), (3.20-21), and $\alpha^{0}=O(1) \alpha^{ \pm}$, we find that the total contribution of the source from $s \leqq t / 8$ is

$$
\begin{aligned}
\int_{0}^{t / 8} \int_{-\infty}^{\infty}\left(I_{1}^{+}+I_{2}^{+}+I_{2}^{-}+I_{2}^{-}\right) d y d s \\
=O(1) e^{-b t} e^{-|x| / 2 C} \int_{0}^{t / 8} \int_{-\infty}^{\infty}\left(1+(t-s)^{1 / 2}\right)(s+1)^{-r} \\
\quad \times K_{\varepsilon}^{ \pm}(x-y, t-s) d y d s \\
=O(1)\left(1+t^{1 / 2}\right) e^{-b t} e^{-e|x| / 2 C} \int_{0}^{t / 8}(s+1)^{-r} d s, \\
=O(1)(t+1)^{1-r}\left(1+t^{1 / 2}\right) e^{-b t} e^{-|x| / 2 C} .
\end{aligned}
$$

This exponentially small term can be absorbed into $(1+t)^{-r+1} e^{-|x| / 2 C}$.

To estimate the contribution from $s \geqq t / 8$, we use only $K_{C}^{ \pm}(x-y, t-s)$ $W_{C}(y, s+1) \leqq \frac{1}{\sqrt{4 \pi C(t-s)}} W_{C}(x, t+1)$. This gives

$$
\begin{aligned}
\int_{t / 8}^{t} \int_{-\infty}^{\infty} I_{1}^{ \pm} d y d s= & O(1) W_{C}(x, t+1) \int_{t / 8}^{t} \int_{-\infty}^{\infty}(s+1)^{-r}(t-s)^{-1 / 2} \\
& \times K_{\varepsilon}^{ \pm}(x-y, t-s) d y d s \\
= & O(1)(t+1)^{-r} W_{C}(x, t+1) \int_{t / 8}^{t}(t-s)^{-1 / 2} d s, \\
= & O(1)(t+1)^{-r+1 / 2} W_{C}(x, t+1),
\end{aligned}
$$


and

$$
\begin{aligned}
\int_{t / 8}^{t} \int_{-\infty}^{\infty} I_{1}^{ \pm} d y d s & =O(1) \alpha^{0}(x) \int_{t / 8}^{t} \int_{-\infty}^{\infty}(s+1)^{-r} K_{\varepsilon}^{ \pm}(x-y, t-s) d y d s \\
& =O(1) \alpha^{0}(x) \int_{t / 8}^{t}(s+1)^{-r} d s \\
& =O(1)(t+1)^{-r+1} e^{-e|x| / 2 C}
\end{aligned}
$$

\section{Lemma 3.1.b. If}

$$
|S|=O(1)(t+1)^{-q} e^{-|x| / 2 C}
$$

then

$$
|u|=O(1)(t+1)^{-q} e^{-|x| / 2 C} .
$$

Proof. Using (2.4) and $K_{x}^{ \pm}(y, s)=O(1) s^{-1 / 2} K_{C}^{ \pm}(y, s)$ we have

$$
|u(x, t)|=\int_{0}^{t} \int_{-\infty}^{\infty}\left(I_{1}^{+}+I_{2}^{+}+I_{1}^{-}+I_{2}^{-}\right) d y d s,
$$

where

$$
\begin{gathered}
I_{1}^{ \pm}=\alpha(x) K_{x}^{ \pm}(x-y, t-s) S(y, s) \\
=O(1)(t-s)^{-1 / 2}(s+1)^{-q} \alpha^{\mp}(x) K_{C}^{ \pm}(x-y, t-s) e^{-|y| / 2 C}, \\
I_{2}^{ \pm}=\mp \alpha^{0}(x) K^{ \pm}(x-y, t-s) S(y, s) \\
=O(1)(s+1)^{-q} \alpha^{0}(x) K^{ \pm}(x-y, t-s) e^{-|y| / 2 C} .
\end{gathered}
$$

From the identity

$$
e^{\frac{-[(x-y) \pm(t-s)]^{2}}{4 C(t-s)}} e^{\mp y / 2 C}=e^{\mp x / 2 C} e^{-(t-s) / 4 C} e^{\frac{-(x-y)^{2}}{4 C(t-s)}},
$$

and the observation

$$
\frac{e^{ \pm x / 2}}{e^{x / 2}+e^{-x / 2}} \leqq \begin{cases}1 & x \gtrless 0 \\ e^{ \pm x} & x \gtrless 0,\end{cases}
$$

it follows that, for $C \geqq 1$,

$$
\frac{e^{ \pm x / 2}}{e^{x / 2}+e^{-x / 2}} e^{\frac{-[(x-y) \pm(t-s)]^{2}}{4 C(t-s)}} e^{-|y| / 2 C}=O(1) e^{-|x| / 2 C} e^{-(t-s) / 4 C} e^{\frac{-(x-y)^{2}}{4 C(t-s)}},
$$

or, in other words, that

$$
\alpha^{\mp}(x) K_{C}^{ \pm}(x-y, t-s) e^{-e|y| / 2 C}=O(1) e^{-|x| / 2 C}(t-s)^{-1 / 2} e^{-\frac{(x-y)^{2}}{4 c(t-s)}} e^{-(t-s) / 4 C} .
$$

Since, also, $\alpha^{0}(x)=O(1) \alpha \mp(x)$, we have

$$
\begin{aligned}
& \left(I_{1}^{+}+I_{2}^{+}+I_{1}^{-}+I_{2}^{-}\right) \\
& \quad=O(1) e^{-|x| / 2 C}\left[(t-s)^{-1 / 2}+(t-s)^{-1}\right](s+1)^{-q} e^{-(t-s) / 4 C} e^{\frac{(x-y)^{2}}{4 C(t-s)}} .
\end{aligned}
$$


This gives

$$
\begin{aligned}
& \int_{0}^{t} \int_{-\infty}^{\infty}\left(I_{1}^{+}+I_{2}^{+}+I_{1}^{-}+I_{2}^{-}\right) d y d s \\
& =O(1) e^{|x| / 2 C} \int_{0}^{t} d s e^{-(t-s) / 4 C}\left[1+(t-s)^{-1 / 2}\right](s+1)^{-q} \\
& \quad \times \int_{-\infty}^{\infty} d y(t-s)^{-1 / 2} e^{-\frac{(x-y)^{2}}{4 C(t-s)}} \\
& =O(1) e^{|x| / 2 C} \int_{0}^{t} e^{-(t-s) / 4 C}\left[1+(t-s)^{-1 / 2}\right](s+1)^{-q} d s \\
& =O(1)(t+1)^{-q} e^{|x| / 2 C} .
\end{aligned}
$$

Proof of Proposition 3.1. (1) The bound on $|u|$ follows directly from Lemmas 3.1.a and 3.1.b.

Differentiating (2.4) and integrating by parts, we have

$$
\left|u_{x}(x, t)\right|=\int_{0}^{-\infty} \int_{-\infty}^{\infty}\left(I_{1}^{+}+I_{2}^{+}+I_{3}^{+}+I_{1}^{-}+I_{2}^{-}+I_{3}^{1}\right) d y d s,
$$

where

$$
\begin{aligned}
& I_{1}^{ \pm}=\alpha \mp(x) K_{x}^{ \pm}(x-y, t-s) S_{x}(y, x), \\
& I_{2}^{ \pm}=\mp 2 \alpha^{0}(x) K^{ \pm}(x-y, t-s) S_{x}(y, s), \\
& I_{3}^{ \pm}= \pm \alpha^{0^{\prime}}(x) K^{ \pm}(x-y, t-s) S(y, s) .
\end{aligned}
$$

Since $\alpha^{0^{\prime}}(x)=O(1) \alpha^{0}(x)$, and $S_{x}=O(1) t^{-1 / 2}(t+1)^{1 / 2} S$, each of these terms in similar to a type already treated in the proofs of the lemmas, and the bound on $\left|u_{x}\right|$ follows from the same calculations.

(ii) Without loss of generality, we may take $|S|=O(1)(t+1)^{-p} K_{C}^{+}(x, t+$ $1)^{2},\left|S_{x}\right|=O(1) t^{-1 / 2}(t+1)^{-p} K_{C}^{+}(x, t+1)^{2}$.

From, $K^{ \pm} x(y, s)=O(1) K_{\varepsilon}^{ \pm}(y, s) K_{C}^{ \pm}(y, s), K^{ \pm}(y, s)=O(1) s^{1 / 2} K_{\varepsilon}^{ \pm}(y, s) K_{C}^{ \pm}(y, s)$, we have

$$
|u(x, t)|=\int_{0}^{t} \int_{-\infty}^{\infty}\left(I_{1}^{+}+I_{2}^{+}+I_{1}^{-}+I_{2}^{-}\right) d y d s
$$

where

$$
\begin{aligned}
I_{1}^{ \pm}= & \alpha \mp(x) K_{x}^{ \pm}(x-y, t-s) S(y, s) \\
= & O(1) \alpha^{\mp}(x) K_{\varepsilon}^{ \pm}(x-y, t-s) K_{C}^{ \pm}(x-y, t-s) \\
& \times(s+1)^{-p} K_{C}^{+}(y, s+1)^{2}, \\
I_{2}^{ \pm}= & \alpha^{0}(x) K^{ \pm}(x-y, t-s) S(y, s) \\
= & O(1) \alpha^{0}(x) K_{\varepsilon}^{ \pm}(x-y, t-s) K_{C}^{ \pm}(x-y, t-s) \\
& \times(t-s)^{1 / 2}(s+1)^{-p} K_{C}^{+}(y, s+1)^{2} .
\end{aligned}
$$

Since $(t+1)^{-p} K_{C}^{+}(x, t+1) \leqq(t+1)^{-p} W_{C}^{+}(x, t+1)$, the calculation in Lemma 3.1.a shows that the contribution from $s \leqq t / 8$ is negligible. 
The "parallel propagation" terms $I_{1}^{+}$and $I_{2}^{+}$in fact give negligible contribution for all of $0 \leqq s \leqq t$. For, identity (3.19) shows that

$$
K_{C}^{+}(x-y, t-s) K_{C}^{+}(y, s+1) \leqq \frac{1}{4 \pi C}(t-s)^{-1 / 2}(s+1)^{-1 / 2}(t+1)^{1 / 2} K_{C}^{+}(x, t+1) .
$$

Thus,

$$
\begin{aligned}
\alpha^{-}(x) & K \varepsilon^{ \pm}(x-y, t-s) K_{C}^{ \pm}(x-y, t-s) K_{C}^{+2}(y, s+1) \\
& =O(1) \alpha^{-}(x)(t+1)(t-s)(s+1) K_{\varepsilon}^{ \pm}(x, t+1) K_{C}^{ \pm}(x, t+1) \\
& =O(1)(t-s)^{-1}(s+1)^{-1}(t+1) e^{-b t} K_{C}^{+}(x, t+1)
\end{aligned}
$$

for $b>0$, and

$$
\begin{aligned}
& \int_{0}^{t} \int_{-\infty}^{\infty}\left(I_{1}^{+}+I_{2}^{+}\right) d y d s \\
& =O(1) e^{-b t}(t+1) K_{C}^{+}(x, t+1) \int_{0}^{t}(t-s)^{-1 / 2}(s+1)^{-p-1}\left(1+(t-s)^{-1 / 2}\right) \\
& \quad \times \int_{-\infty}^{\infty} K_{\varepsilon}^{+}(x, t+1) d y d s \\
& =O(1) e^{-b t}(t+1) K_{C}^{+}(x, t+1) \int_{0}^{t} 1+(t-s)^{-1 / 2}(t-s)^{-1 / 2}(s+1)-p-1 d s \\
& =O(1) e^{-b t} K_{C}^{+}(x, t+1) .
\end{aligned}
$$

The "transverse propagation" terms $I_{1}^{-}$and $I_{2}^{-}$are the critical terms, and their estimation uses in a crucial way the transversality of the propagator $K^{-}$and the source $K^{+}$, via the identity

$$
\begin{aligned}
\int_{0}^{t} \int_{-\infty}^{\infty} K_{C}^{-}(x-y, t-s) K_{C}^{+}(y, s+1) d y d s \\
\quad=\int_{-\infty}^{x} 1 / 2\left[K_{C}^{+}(y, t+1)-K_{C}^{-}(y+2, t+1)\right] d y \\
\quad \leqq 1
\end{aligned}
$$

The easiest way to verify this identity is to notice that both terms, when considered as functions of $(x, t)$, satisfy the equation

$$
w_{t}-w_{x}-C w_{x x}=K^{+}(x, t+1) ; \quad w(x, 0) \equiv 0,
$$

the first by Duhamel's principle and the second by direct substitution.

We find that

$$
\begin{aligned}
\int_{t / 8}^{t} \int_{-\infty}^{\infty} I_{2}^{-} d y d s= & O(1) \alpha^{0}(x) \sup _{y, s \geqq t / 8}\left|K_{C}^{+}(y, s+1)\right|(s+1)^{-p} \\
& \times \int_{0}^{t} \int_{-\infty}^{\infty} K_{C}^{-}(x-y, t-s) K_{C}^{+}(y, s+1) d y d s \\
= & O(1)(t+1)^{-p-1 / 2} e^{-|x| / 2 C}
\end{aligned}
$$

as required. 
Finally, integrating by parts, we obtain

$$
\begin{aligned}
\int_{t / 8}^{t} \int_{-\infty}^{\infty} I_{1}^{-} d y d s= & \alpha^{+}(x) \int_{t / 8}^{t} \int_{-\infty}^{\infty} K_{x}^{-}(x-y, t-s) S(y, s) d y d s \\
= & \alpha^{+}(x) \int_{t / 8}^{t} \int_{-\infty}^{\infty} K^{-}(x-y, t-s) S_{x}(y, s) d y d s \\
= & O(1) \int_{t / 8}^{t} \int_{-\infty}^{\infty} s^{-1 / 2}(x+1)^{-p}(t-s)^{1 / 2} \\
& \times K_{C}^{-}(x-y, t-s) K_{\varepsilon}^{-}(x-y, t-s) K_{C}^{+}(y, s+1)^{2} d y d s .
\end{aligned}
$$

Using identities (3.20) and (3.46), we obtain

$$
\begin{aligned}
\int_{t / 8}^{t} \int_{-\infty}^{\infty} I_{1}^{-} d y d s= & O(1) W_{C}(x, t+1) \sup _{y, s \geqq t / 8}\left|K_{C}^{+}(y, s+1)\right|(s+1)^{-p-1 / 2} \\
& \times \int_{0}^{t} \int_{-\infty}^{\infty} K_{\varepsilon}^{-}(x-y, t-s) K_{\varepsilon}^{+}(y, s+1) d y d s \\
= & O(1) t^{-1 / 2}(t+1)^{-p-1 / 2} W_{C}(x, t+1) .
\end{aligned}
$$

The same calculation without integration by parts gives $O(1)(t+1)^{-p-1 / 2}$ $W_{C}(x, t+1)$. Thus, interpolation completes the estimate for $|u|$.

The estimate for $\left|u_{x}\right|$ is not critical, and follows from the same calculations used in the proof of Lemma 3.1.a together with the crude bound $K_{C}(x, t)=$ $O(1) t^{-1 / 2} W_{C}(x, t)$. This completes the proof of Proposition 3.1.

Proof of Proposition 3.2. By symmetry, we need treat only source terms involving $K_{C}^{+}$.

We start with the weaker hypothesis,

$$
\begin{aligned}
|S| & =O(1)(t+1)^{-1 / 2} K_{C}^{+}(x, t+1), \\
\left|S_{x}\right| & =O(1) t^{-1 / 2} K_{C}^{+}(x, t+1),
\end{aligned}
$$

which is valid in both case (i) and (ii).

After integrating (2.5) by parts, we have

$$
|u(x, t)|=O(1) \int_{0}^{t} \int_{-\infty}^{\infty}\left(I_{1}^{+}+I_{2}^{+}+I_{1}^{-}+I_{1}^{-}\right) d y d s,
$$

where

$$
\begin{aligned}
I_{1}^{ \pm} & =\alpha^{ \pm}(y) K_{x}^{ \pm}(x-y, t-s) S(y, s) \\
& =O(1)(t-s)^{-1 / 2} \alpha^{ \pm}(y) K_{C}^{ \pm}(x-y, t-s)(s+1)^{-1 / 2} K_{c}^{+}(y, s+1) \\
I_{2}^{ \pm} & = \pm \alpha^{0}(y) K^{ \pm}(x-y, t-s) S(y, s) \\
& =O(1) \alpha^{0}(y) K^{ \pm}(x-y, t-s)(s+1)^{-1 / 2} K_{C}^{+}(y, s+1)
\end{aligned}
$$

and $\bar{C}<C$. 
For $C>\bar{C}$, we improve identity (3.19) to

$$
\begin{aligned}
& e^{\frac{-(x-y)^{2}}{4 C(t-s)}} e^{-\frac{y^{2}}{4 C(s+1)}} \\
& \quad=e^{\left[-\frac{x^{2}}{4 C(t+1)}-\frac{(C / \bar{C}-1)(t-s)}{4(t+1)[(t+1)+(C / C-1)(s+1)]} x^{2}-\frac{(t+1)+(C / \bar{C}-1)(s+1)}{4 C(t-s)(s+1)}\left(y-\frac{C / C \bar{C}(s+1)}{(t+1)+(C / \bar{C}-1)(s+1)} x\right)^{2}\right]} .
\end{aligned}
$$

The change of coordinates $x^{\prime}=x-(t+1)$, gives $y^{\prime}=y-(s+1)$, gives

$$
\begin{aligned}
& K_{\bar{C}}^{+}(x-y, t-s) K_{C}^{+}(y, s+1) \\
& \quad=K_{C}^{+}(x, t+1)(t+1)^{1 / 2}(t-s)^{-1 / 2}(s+1)^{-1 / 2} \beta(s, y, t, s),
\end{aligned}
$$

where

$$
\begin{aligned}
\beta(x, y, t, s)= & e^{-\frac{(c / \bar{C}-1)(t-s)}{r(t+1)[(t+1)+(C / \bar{C}-1)(s+1)]}[x-(t+1)]^{2}} \\
& \times e^{-\frac{(t+1)+(C / \bar{C}-1)(s+1)}{4 C(t-s)(s+1)}\left[(y-s-1)-\frac{C / \bar{C}(s+1)}{(t+1)+(C / \bar{C}-1)(s+1)}(x-g-1)\right]^{2}} .
\end{aligned}
$$

The function $\beta(x, y, t, x)$ is localized around $(x, y)=(t+1, s+1)$. This motivates

Lemma 3.2.a. For $\eta>0$,

$$
e^{-\eta|y|} \beta(x, y, t, s)=O(1) e^{-b(s+1)(t-s) /(t+1)},
$$

where $b>0$ is independent of $t$.

Proof. Making the change of variables $x^{\prime}=\frac{x-(t+1)}{(t+1)^{1 / 2}(s+1)^{1 / 2}}, y^{\prime}=\frac{y(t+1)}{(s+1)(t-s)}$ we have $e^{-\eta|y|} \beta(x, y, t, s)=e^{-\hat{b}(s+1)(t-s) /(t+1)}$, where

$$
\begin{aligned}
\hat{b}= & (1 / 4 C)(C / \bar{C}-1) x^{\prime 2}+\eta\left|y^{\prime}\right| \\
& +1 / 4 C(s+1)^{2}\left[-(s+1)+\frac{(s+1)(t-s)}{(t+1)} y^{\prime}+\frac{C / \bar{C}(s+1)^{3 / 2}(t+1)^{1 / 2}}{(t+1)+(C / \bar{C}-1)(s+1)} x^{\prime}\right]^{2}
\end{aligned}
$$

Either $\left|x^{\prime}\right|>\varepsilon$ or $\left|y^{\prime}\right|>\varepsilon$ or else $\left|\frac{(s+1)(t-2)}{(t+1)} y^{\prime}+\frac{C / \bar{C}(s+1)^{3 / 2}(t+1)^{1 / 2}}{(t+1)+(C / \bar{C}-1)(s+1)} x^{\prime}\right| \leqq \varepsilon(C / \bar{C}+$ $1)(s+1)$, so that $\left[1 / 4 C(s+1)^{2}\right]\left[-(s+1)+\frac{(s+1)(t-s)}{(t+1)} y^{\prime}+\frac{C / \bar{C}(s+1)^{3 / 2}(t+1)^{1 / 2}}{(t+1)+(C / \bar{C}-1)(s+1)} x^{\prime}\right]^{2}>$ $(1 / 4 C)[1-\varepsilon(C / \bar{C}+1)]^{2}$. Thus, $\hat{b} \geqq b>0$, proving the claim.

Combining (3.54), $(3,56)$, and the Lemma 3.2a, we have

$$
I_{2}^{+}=O(1) K_{C}^{+}(x, t+1)(t+1)^{1 / 2}(t-s)^{-1 / 2}(s+1)^{-1} e^{-b(s+1)(t-s) /(t+1)} e^{-|y| / 2} .
$$


Therefore,

$$
\begin{aligned}
\int_{0}^{t} \int_{-\infty}^{\infty} I_{2}^{+} d y d s= & (t+1)^{1 / 2} K_{C}^{+}(x, t+1)\left[\int_{0}^{t} \int_{-\infty}^{\infty}(t-s)^{-1 / 2}(s+1)^{-1}\right. \\
& \left.\times e^{-b(s+1)(t-s) /(t+1)} e^{-|y| / 2}\right] d y d s \\
= & O(1) K_{C}^{+}(x, t+1)(t+1)^{1 / 2} \\
& \times \int_{0}^{t}(t-s)^{1 / 2}(s+1)^{-1} e^{-b(s+1)(t-s) /(t+1)} d s \\
= & O(1) K_{C}^{+}(x, t+1)
\end{aligned}
$$

as claimed.

Likewise, we have

$$
I_{1}^{+}=O(1) K_{C}^{+}(x, t+1)(t+1)^{1 / 2}(t-s)^{-1}(s+s)^{-1} \alpha^{+}(y) \beta(s, y, t, s) .
$$

Using $\alpha^{+}(y) \leqq 1$ and $\beta(x, y, t, s) \leqq e^{-\frac{(t+1)+(C / 2-1(s+1)}{4 C(t-s)(s+)}\left[(y-s-1)-\frac{C / 2(s+1)}{(t+1)+(C / 2-1)(s+1)}(x-t-t)\right]^{2}}$, we have

$$
\begin{aligned}
\int_{0}^{t} \int_{-\infty}^{\infty} I_{1}^{+} d y d s= & (t+1)^{1 / 2} K_{C}^{+}(x, t+1) \int_{0}^{t} \int_{-\infty}^{\infty}(t-s)^{-1}(s+1)^{-1} \\
& \times e^{-\frac{(t+1)+(C / 2-1)(s+1)}{4 C(t-s)(s+1)}\left[(y-s-1)-\frac{C / 2(s+1)}{(t+1)+(C / 2-1)(s+1)}(x-t-1)\right]^{2}} d y d s \\
= & O(1) K_{C}^{+}(x, t+1)(t+1)^{1 / 2} \\
& \times \int_{0}^{t}(t-s)^{-1}(s+1)^{-1}\left(\frac{4 C(t-s)(s+1)}{(t+1)+(C / s-1)(s+1)}\right)^{1 / 2} d s \\
= & O(1) K_{C}^{+}(x, t+1) \int_{0}^{t}(t-s)^{-1 / 2}(s+1)^{-1 / 2} d s \\
= & O(1) K_{C}^{+}(x, t+1),
\end{aligned}
$$

as claimed

The remaining, "transverse propagation" terms,

$$
\begin{aligned}
& I_{1}^{-}=O(1)(t-s)^{-1 / 2} \alpha^{-}(y) K_{x}^{-}(x-y, t-s)(s+1)^{-1 / 2} K_{C}^{+}(y, s+1), \\
& I_{2}^{-}=O(1) \alpha^{0}(y) K^{-}(x-y, t-s)(s+1)^{-1 / 2} K_{C}^{+}(y, s+1)
\end{aligned}
$$

can be treated in a symmetric fashion, since

$$
\begin{gathered}
\alpha^{-}(y) K_{C}^{+}(y, s+1) \leqq \alpha^{+}(y) K_{C}^{-}(y, s+1) \text { and } \\
\alpha^{0}(y) K_{C}^{+}(y, s+1) \leqq e^{-\frac{(C-1}{C)}|y|} K_{C}^{-}(y, s+1),
\end{gathered}
$$

for $C \geqq 1$. This establishes the desired bound for $|v|$.

Differentiating (2.5), we obtain

$$
\left|v_{x}(x, t)\right|=\int_{0}^{t} \int_{-\infty}^{\infty}\left(I^{+}+I^{-}\right) d y d s
$$


where

$$
I^{ \pm}=\alpha^{ \pm}(y) K_{x}^{ \pm}(x-y, t-s) S_{x}(y, s) .
$$

Integrating by parts gives the alternate formulation

$$
\left|v_{x}(x, t)\right|=\int_{0}^{t} \int_{-\infty}^{\infty}\left(I_{1}^{+}+I_{2}^{+}+I_{1}^{-}+I_{2}^{-}\right) d y d s
$$

where

$$
\begin{aligned}
& I_{1}^{ \pm}=\alpha^{ \pm}(y) K_{x x}^{ \pm}(x-y, t-s) S(y, s), \\
& I_{2}^{ \pm}=\mp \alpha^{0}(y) K_{x}^{ \pm}(x-y, t-s) S(y, s) .
\end{aligned}
$$
that

Using $S_{x}=O(1)(t)^{-1 / 2} K_{C}^{+}(x, t+1)$ and the same calculations as above, we find

$$
\begin{aligned}
\left|v_{x}(x, t)\right|= & \int_{t-1}^{t} \int_{-\infty}^{\infty}\left(i^{+}+I^{-} d y d s,+\int_{0}^{t-1} \int_{-\infty}^{\infty}\left(I_{1}^{+}+I_{1}^{-}\right) d y d s\right. \\
& +\int_{0}^{t-1} \int_{-\infty}^{\infty}\left(I_{2}^{+}+I_{2}^{-}\right) d y d s \\
= & O(1)\left[K_{C}^{+}(x, t+1)(t+1)^{1 / 2} \int_{t-1}^{t}(t-s)^{-1 / 2}(s)^{-1 / 2}(s+1)^{-1 / 2} d s\right. \\
& +K_{C}^{+}(x, t+1) \int_{0}^{t-1}(t-s)^{-1}(s+1)^{-1 / 2} d s \\
& \left.\left.+K_{C}^{+}(x, t+1)(t+1)^{1 / 2} \int_{0}^{t-1}(t-s)^{-1}\right)(s+1)^{-1} e^{-b(s+1)(t-s) /(t+1)} d s\right] \\
= & O(1) K_{C}^{+}(x, t+1)\left[(t+1)^{-1 / 2}+(t+1)^{-1 / 2} \log (t+1)+(t+1)^{-1 / 2}\right] .
\end{aligned}
$$

The $\log (t+1)$ contribution of the critical term $\int_{t / 2}^{t-1} \int_{-\infty}^{+\infty}\left(I_{1}^{+}+I_{1}^{-}\right) d y d s$ is all that prevents our claims for $\left|v_{x}\right|$. To complete the proof of Proposition 3.2, we improve the estimate on this critical term by using the extra hypotheses of (i) and (ii), respectively:

In case (i), we have

$$
|S(x, t)|=O(1)\left[(t+1)^{-1 / 2} e^{-|x| / 2 C}+(t+1)^{-1}\right] K_{C}^{+}(x, t+1),
$$

which we estimate term by term.

For $|S|=O(1)(t+1)^{-1} K_{C}^{+}(x, t+1)$, the critical estimate becomes

$$
K_{C}^{+}(x, t+1)(t+1)^{1 / 2} \int_{t / 2}^{t-1}(t-s)^{-1}(s+1)^{-3 / 2} d s=(t+1)^{-1} \log (t) K_{C}^{+}(x, t+1),
$$

which is now satisfactory.

For $|S|=O(1)(t+1)^{-1 / 2} e^{-|x| / 2 C} K_{C}^{+}(x, t+1)$, we recall that Lemma 3.2.a gives $\beta(x, y, t, s) e^{-|y| / 2 C} \leqq e^{-b^{\prime}(t-s)(s+1) /(t+1)}$ for some $b^{\prime}>0$ independent of $t$. Thus, the 
critical estimate becomes

$$
\begin{aligned}
O(1)(t+1)^{1 / 2} & K_{C}^{+}(x, t+1) \int_{t / 2}^{t-1} \int_{-\infty}^{\infty}(t-s)^{-3 / 2}(s+1)^{-1} \\
& \times e^{-b^{\prime}(t-s)(s+1) /(t+1)} e^{-|u| / w C} d y d s \\
= & O(1) K_{C}^{+}(x, t+1)(t+1)^{1 / 2} \int_{0}^{t-1}(t-s)^{-3 / 2}(s+1)^{-1} \\
& \times e^{-b^{\prime}(t-s)(s+1) /(t+1)} d s \\
= & O(1) K_{C}^{+}(x, t+1)(t+1)^{-1 / 2},
\end{aligned}
$$

which again is satisfactory.

Case (ii) follows immediately from (3.15), since the contribution on $t / 2 \leqq s \leqq$ $t-1$ can be handled using (3.69-70) and the same calculations used to estimate $|v|$.

This completes the proof of Proposition 3.2

Remark. The analysis is also sufficient to treat the genuinely nonlinear case including a $v^{2}$ term in the flux of $v$. Proposition 3.2(ii) would then be necesary, and is included for that reason. It is not used in the analysis of the complex Burgers equation.

\section{Stability of the Solution}

We now consider the full nonlinear perturbation equations

$$
\begin{aligned}
& L_{1}(u)=1 / 2\left(u^{2}-v^{2}\right)_{x} ; \quad u(x, 0)=U^{0}(x), \\
& L_{2}(v)=(-u v)_{x} ; \quad v(x, 0)=v^{0}(x)
\end{aligned}
$$

with a localized perturbation.

Our basic stability result is contained in the following proposition, which corresponds to $L^{1}$-bounded stability. We take $t_{0}=1$ for simplicity.

Proposition 4.1. Let $C>1$ and let $\left|u^{0}(x)\right|,\left|v^{0}(x)\right| \leqq \delta e^{-x^{2} / 4}$ and $\int_{-\infty}^{\infty} u^{0}(x) d x=0$. For $\delta$ sufficiently small, (4.1) has a global solution satisfying

$$
\begin{aligned}
u(x, t) & =O(1) \delta\left[(t+1)^{-1} W_{C}(x, t+1)+(t+1)^{-1 / 2} e^{-|x| / 2 C}\right], \\
u_{x}(x, t) & =O(1) \delta\left[t^{-1 / 2}(t+1)^{-1 / 2} W_{C}(x, t+1)+(t+1)^{-1 / 2} e^{-|x| / 2 C}\right], \\
v(x, t) & =O(1) \delta\left[K_{C}^{-}(x, t+1)+K_{C}^{+}(x, t+1)\right], \\
v_{x}(x, t) & =O(1) \delta\left[(t)^{-/ 12} K_{C}^{-}(x, t+1)+K_{C}^{+}(x, t+1)\right],
\end{aligned}
$$

where $O(1)$ is a bound depending only on $C$.

Proof. We proceed by iteration. Let $\left\{u_{k}, v_{k}\right\}$ be the sequence defined by

$$
\begin{aligned}
u_{0}(x, t) & =v_{0}(x, t)=0, \\
L_{1}\left(u_{k+1}\right) & =1 / 2\left(u_{k}^{2}-v_{k}^{2}\right)_{x} ; \quad u_{k+1}(x, 0)=u^{0}(x), \\
L_{2}\left(v_{k+1}\right) & =\left(-u_{k} v_{k}\right)_{x} ; \quad v_{k+1}(x, 0)=v^{0}(x),
\end{aligned}
$$


Short time existence theory (cf. $[\mathrm{H}-\mathrm{Sm}]$ ) guarantees that the iterates $\left\{u_{k}, v_{k}\right\}$, converge to a global solution of (4.1), provided that $\left|u_{k}\right|$ and $\left|v_{k}\right|$ are uniformly bounded.

Lemma 4.1.a A Priori Estimate. The sequence $\left\{u_{k}, v_{k}\right\}$ satisfies

$$
\begin{aligned}
u_{k}(x, t) & \leqq \delta_{k}\left[(t+1)^{-1} W_{C}(x, t+1)+(t+1)^{-1 / 2} e^{-|x| / 2 C}\right], \\
u_{k_{x}}(x, t) & \leqq \delta_{k}\left[t^{-1 / 2}(t+1)^{-1 / 2} W_{C}(x, t+1)+(t+1)^{-1 / 2} e^{-|x| / 2 C}\right], \\
v(x, t) & \leqq \delta_{k}\left[K_{C}^{-}(x, t+1)+K_{C}^{+}(x, t+1)\right], \\
v_{k_{x}}(x, t) & \leqq \delta_{k}(t)^{-1 / 2}\left[K_{C}^{-}(x, t+1)+K_{C}^{+}(x, t+1)\right],
\end{aligned}
$$

where $\delta_{0}=0$ and $\delta_{k+1}=O(1)\left(\delta+\delta_{k}^{2}\right)$, where $O(1)$ is a bound depending only on $C$.

Proof. We proceed by induction, noting that the assertion holds trivially for $k=0$.

We can write $u_{k}=\hat{u}_{k}+\hat{\hat{u}}_{k}, v_{k}=\hat{v}_{k}+\hat{\hat{v}}_{k}$, where

$$
\begin{aligned}
& L_{1}\left(\hat{u}_{k+1}\right)=0 ; \quad \hat{u}_{k+1}(x, 0)=u^{0}(x), \\
& L_{1}\left(\hat{\hat{u}}_{k+1}\right)=1 / 2\left(u_{k}^{2}-v_{k}^{2}\right)_{x} ; \quad \hat{\hat{u}}_{k+1}(x, 0)=0, \\
& L_{2}\left(\hat{v}_{k+1}\right)=0 ; \quad \hat{v}_{k+1}(x, 0)=v^{0}(x), \\
& L_{2}\left(\hat{\hat{u}}_{k+1}\right)=\left(v_{k}^{2}-v_{k}^{2}\right)_{x} ; \quad \hat{\hat{u}}_{k+1}(x, 0)=0 .
\end{aligned}
$$

By the induction hypothesis, the source terms $S_{1}=\left(u_{k}^{2}-v_{k}^{2}\right)$ and $S_{2}=\left(u_{k} v_{k}\right)$ satisfy

$$
\begin{aligned}
S_{1}= & O(1) \delta_{k}^{2}\left[(t+1)^{-2} W_{C}(x, t+1)^{2}+(t+1)^{-1} e^{-|x| / 2 C}\right. \\
& \left.+K_{C}^{-}(x, t+1)^{2}+K_{C}^{+}(x, t+1)^{2}\right], \\
S_{1_{x}}= & O(1) \delta_{k}^{2}\left[(t+1)^{-2} W_{C}(x, t+1)^{2}+(t+1)^{-1} e^{-|x| / 2 C}\right. \\
& \left.+(t+1)^{-1 / 2}\left(K_{C}^{-}(x, t+1)^{2}+K_{C}^{+}(x, t+1)^{2}\right)\right],
\end{aligned}
$$

and

$$
\begin{aligned}
S_{2}= & O(1) \delta_{k}^{2}\left[(t+1)^{-1 / 2} e^{-|x| / C}+(t+1)^{-1}\right]\left[K_{C}^{-}(x, t+1)\right. \\
& \left.+K_{C}^{+}(x, t+1)\right] \\
S_{2_{x}}= & O(1) \delta_{k}^{2} t^{-1 / 2}\left|S_{x}\right| \\
& =O(1) t^{-1 / 2}\left[K_{C}^{-}(x, t+1)+K_{C}^{+}(x, t+1)\right] .
\end{aligned}
$$

By Propositions 3.1 and 3.2, and linearity, we find that

$$
\begin{aligned}
\hat{\hat{u}}_{k+1}(x, t) & =O(1) \delta_{k}^{2}\left[(t+1)^{-1} W_{C}(x, t+1)+(t+1)^{-1 / 2} e^{-|x| / 2 C}\right], \\
\hat{\hat{u}}_{k+1_{x}}(x, t) & =O(1) \delta_{k}^{2}\left[t^{-1 / 2}(t+1)^{-1 / 2} W_{C}(x, t+1)+(t+1)^{-1 / 2} e^{-|x| / 2 C}\right], \\
\hat{\hat{v}}_{k+1}(x, t) & =O(1) \delta_{k}^{2}\left[K_{C}^{-}(x, t+1)+K_{C}^{+}(x, t+1),\right. \\
\hat{\hat{v}}_{k+1_{x}}(x, t) & =O(1) \delta_{k}^{2}\left[(t)^{-1 / 2}\left[K_{C}^{-}(x, t+1)+K_{C}^{+}(x, t+1)\right],\right.
\end{aligned}
$$

as claimed. 
From Proposition 2.1, we have

$$
\begin{aligned}
& \hat{u}(x, t)=\int_{-\infty}^{\infty} U(y, 0) G_{1}(x, t ; y) d y, \\
& \hat{v}(x, t)=\int_{-\infty}^{\infty} v(y, 0) G_{2}(x, t ; y) d y .
\end{aligned}
$$

By hypothesis, $u^{0}(x), v^{0}(x)=O(1) \delta K^{ \pm}(x, 1)$. The condition

$$
\int_{-\infty}^{\infty} u(y, 0) d y=0
$$

implies that $U^{0}(x)+\int_{-\infty}^{x} u^{0}(y) d y=\int_{x}^{+\infty}(y) d y$ is bounded by $O(1) \delta K^{ \pm}(x, 1)$, as well, since

$$
\begin{aligned}
U^{0}(x) & =\int_{x}^{\infty} u^{0}(y) d y \\
& \leqq \int_{x}^{\infty} k^{ \pm}(y, 1) d y \\
& =O(1) K \pm(|x|, 1),
\end{aligned}
$$

for $x \geqq 1$, and similarly for $x \leqq-1$.

We thus have

$$
\begin{aligned}
\hat{u}= & O(1) \delta\left[\int_{-\infty}^{\infty} \alpha \mp(x) K^{ \pm}(x-y, t) K^{ \pm}(y, 1) d y,\right. \\
& +\alpha^{0}(x) \int_{-\infty}^{\infty} K^{ \pm}(x-y, t) K^{ \pm}(y, 1) d y,
\end{aligned}
$$

where we have integrated by parts to replace terms $\alpha^{\mp}(x) K_{x}^{ \pm}(x-y, t) U^{0}(y)$ by terms of form $\alpha^{\mp}(x) K^{ \pm}(x-y, t) u^{0}(y)$.

Since

$$
\int_{-\infty}^{\infty} K^{ \pm}(x-y, t) K^{ \pm}(y, 1)=K^{ \pm}(x, t+1)
$$

(by the semigroup property of the heat equation), and $\alpha^{0}=O(1) \alpha^{ \pm}$, we conclude that

$$
\begin{aligned}
\hat{u} & =O(1) \delta \alpha^{\mp}(x) K^{ \pm}(x, t+1) \\
& =O(1) \delta e^{-b t} K_{C}^{ \pm}(x, t)
\end{aligned}
$$

for some $b>0$, as claimed.

The bound on $\hat{u}_{x}$ follows similarly, as do the bounds on $\hat{v}$ and $\hat{v}_{x}$.

From the lemma, we have $\delta_{k+1} \leqq M\left(\delta+\delta_{k}^{2}\right)$, for all $k$. Thus, if $4 \delta M^{2} \leqq 1$, it follows by induction that $\delta_{k} \leqq 2 M \delta$ for all $k$. From this uniform bound, we obtain both convergence of the iteration scheme, and, in the limit, the desired bounds on the solution. 


\section{Proof of Main Theorems}

We can obtain complete $L^{1}$ information using the basic stability result and different bootstrap arguments.

Proof of Theorem 1. Without loss of generality, we restrict to the case $t_{0}=1$ treated in Proposition 4.1. We need to slightly improve our estimates for $u$ and $u_{x}$. We demonstrate the method for $u$, noting that $u_{x}$ follows similarly.

We first observe that the constant $C$ in the estimates of Proposition 4.1 is not sharp, and could be improved to $\bar{C}=(1 / 2)(C+1)$.

We write $u=\hat{u}+\hat{\hat{u}}+\hat{\hat{u}}$, where

$$
\begin{gathered}
L_{1}(\hat{u})=0 ; \quad \hat{u}(x, 0)=u^{0}(x), \\
L_{1}(\hat{\hat{u}})=\left(-v^{2}\right) x ; \quad \hat{\hat{u}}(x, 0)=0, \\
L_{1}(\hat{\hat{\hat{u}}})=\left(-u^{2}\right) x ; \quad \hat{\hat{u}}(x, 0)=0 .
\end{gathered}
$$

The term $\hat{u}$ is exponentially small, as calculated in Sect. 4. Combining Propositions 3.1 (i) and 4.1, we find that $\hat{\hat{u}}$ also satisfies the cliamed bound. However, Proposition 3.1 (ii) is not sufficient to bound the critical term $\hat{\hat{u}}$.

For a better estimate of $\hat{\hat{u}}$, we decompose $v$ into $v=v^{-}+v^{+}$, where

$$
\begin{aligned}
u^{ \pm}(x, t)= & \int_{-\infty}^{\infty} v(y, 0) \alpha^{ \pm}(y) K^{ \pm}(x, t ; y) d y \\
& +\int_{0}^{t} \int_{-\infty}^{\infty} K^{ \pm}(x-y, t-s) \alpha^{ \pm}(y)(u v)_{x}(y, t) d y d s .
\end{aligned}
$$

Without loss of generality, we need consider only the source term $\left(v^{+}\right)_{x}^{2}$ in estimating $\hat{\hat{u}}$, since the term $\left(v^{-}\right)_{x}^{2}$ is a symmetric case, and the cross term $v^{-} v^{+}=K_{\bar{C}}^{+} K_{\bar{C}}^{-}$ is $O(1) e^{-b t} K_{C}^{+}$for some $b>0$, hence negligible (Proposition 3.2).

By the standard solution formula for the heat equation, $v^{+}$satisfies

$$
v_{t}^{+}+v_{x}^{+}-v_{x x}^{+}=\alpha^{+}(x) u v_{x}(x, t),
$$

so that $\theta \triangleq v^{+2}$ satisfies the equation

$$
\theta_{t}+\theta_{x}-\theta_{x x}=\left(v_{x}\right)^{2}+\alpha(x) v(u v)_{x} .
$$

As in the proof of 3.1(ii), parallel propagation of the source $\theta_{x}$ is negligible, as are source effects originating from time $s \leqq t / 8$, and all terms involving $\alpha^{0}(x)$. Thus, our calculation reduces to the estimation of the critical term

$$
\mathscr{I}=\alpha^{+}(x) \int_{t / 8}^{t} \int_{-\infty}^{\infty} K^{-}(x-y, t-s)(\theta)_{x} d y d s .
$$

Lemma 5.1. For fixed $\tau$,

$$
\omega(x, t) \triangleq \int_{\tau}^{t} \int_{-\infty}^{\infty} K^{-}(x-y, t-s)(\theta)_{x} d y d s
$$

satisfies

$$
\omega(x, t)=1 / 2 \theta(x, t)+O(1)\left[(\tau+1)^{-1 / 2} K_{\bar{C}}^{-}(x, t)+(\tau+1)^{-3 / 2} W_{C}(x, t+1)\right] .
$$


Proof. The function $\omega$ satisfies

$$
\begin{aligned}
\omega_{t}-\omega_{x}-\omega_{x x} & =(\theta)_{x}, \\
\omega(x, \tau) & \equiv 0,
\end{aligned}
$$

again by the standard solution formula for the heat equation. By (5.6), the residual

$$
r(x, t) \triangleq \omega(x, t)-(1 / 2) \theta(x, t)
$$

satisifes

$$
\begin{aligned}
r_{t}-r_{x}-r_{x x} & =\left(v_{x}\right)^{2}+\alpha^{+}(x) v(u v)_{x}, \\
r(x, \tau) & =-(1 / 2) \theta(x, \tau),
\end{aligned}
$$

so that

$$
\begin{aligned}
r(x, t) & =\int_{-\infty}^{\infty}(1 / 2) \theta(y, \tau) K^{-}(x-y, t-\tau) d y \\
& +\alpha^{+}(x) \int_{\tau}^{t} \int_{-\infty}^{\infty} K^{-}(x-y, t-s)\left[\left(v_{x}^{+}\right)^{2}+\alpha^{+}(x) v(u v)_{x}\right](y, s) d y d s .
\end{aligned}
$$

The term

$$
\int_{-\infty}^{\infty}(1 / 2) \theta(y, \tau) K^{-}(x-y, t-\tau) d y
$$

is $O(1)(\tau+1)^{-/ 2} K_{\bar{C}}^{-}(x, t+1)$, by a calculation like that of (4.18). The terms

$$
\alpha^{+}(x) \int_{\tau}^{t} \int_{-\infty}^{\infty} K^{-}(x-y, t-s)\left(v_{x}^{+}\right)^{2}(y, s) d y d s
$$

and

$$
\alpha^{+}(x) \int_{\tau}^{t} \int_{-\infty}^{\infty} K^{-}(x-y, t-s) v(u v)_{x}(y, s) d y d s
$$

can be estimated by calculations as in Proposition 3.1(ii) and 3.1(i), respectively, to give

$$
r(x, t)=O(1)\left[K_{\bar{C}}^{-}(x, t)+(\tau+1)^{-3 / 2} W_{C}(x, t+1)\right] .
$$

Setting $\tau=t / 8$ in the lemma, and using $\alpha^{+}(x) K_{\bar{C}}^{-}(x, t+1)=O(1) e^{-b t} W_{C}(x, t+$ 1), $\theta=O(1) K^{+}(x, t+1)^{2}$, we have

$$
\left.\mathscr{I}=O(1) \alpha^{+}(x) p K_{C}^{+2}(x, t+1)+(t+1)^{-3 / 2} W_{C}(x, t+1)\right],
$$

and we are done.

Proof of Theorem 2. The assertion for $u$ follows from Theorem 1.

We write $v=\hat{v}+\hat{\hat{v}}$, where

$$
\begin{aligned}
& L_{2}(\hat{v})=0 ; \quad \hat{v}(x, \tau)=v(x, \tau), \\
& L_{2}(\hat{\hat{v}})=(u v)_{x} ; \quad \hat{v}(x, \tau)=0 .
\end{aligned}
$$


The term $\hat{v}$ can be split into $\hat{v}=\hat{v}^{-}+\hat{v}^{+}$, where

$$
\begin{aligned}
& \hat{v}^{-}(x, t) \triangleq \int_{-\infty}^{\infty} K^{-}(x-y, t-\tau) \alpha^{-}(y) v(y, \tau) d y, \\
& \hat{v}^{+}(x, t) \triangleq \int_{-\infty}^{\infty} K^{+}(x-y, t-\tau) \alpha^{+}(y) v(y, \tau) d y .
\end{aligned}
$$

These terms satisfy convected heat equations

$$
\begin{gathered}
u_{t}^{-}+v_{x}^{-}-v_{x x}^{-}=0 ; \quad v^{-}(x, \tau)=\alpha^{-}(x) v(x, \tau), \\
u_{t}^{+}+v_{x}^{-} v_{x x}^{+}=0 ; \quad v^{+}(x, \tau)=\alpha^{+}(x) v(x, \tau) .
\end{gathered}
$$

Thus, defining

$$
\rho_{\tau}^{ \pm}=\int_{-\infty}^{\infty} \alpha^{ \pm}(y) v(y, \tau) d y
$$

we have (cf.[Che-L], [Zu.2])

$$
\begin{aligned}
& \left\|v^{+}(x, t)-\rho_{\tau}^{+} K^{+}(x, t)\right\| L^{1} \leqq(t-\tau)^{-1 / 2}\left\|(x-\tau) \alpha^{+}(x) v(x, \tau)\right\|_{L^{1}}, \\
& \left\|v^{-}(x, t)-\rho_{\tau}^{-} K^{-}(x, t)\right\|_{L^{1}} \leqq(t-\tau)^{-1 / 2}\left\|(x-\tau) \alpha^{-}(x) v(x, \tau)\right\|_{L^{1}},
\end{aligned}
$$

Since $\alpha^{ \pm} v(x, \tau)=O(1) K_{C}^{ \pm}(x, \tau)$, we have

$$
\int_{-\infty}^{\infty}|x \mp \tau| K_{C}^{ \pm}(x, \tau)=O(1) \tau^{1 / 2}
$$

so that

$$
\begin{aligned}
\left\|v^{+}(x, t)-\rho^{+} K+(x, t)\right\|_{L^{1}} & =O(1) \tau^{1 / 2}(t-\tau)^{-1 / 2}, \\
\left\|v^{-}(x, t)-\rho^{-} K+(x, t)\right\|_{L^{1}} & =O(1) \tau^{1 / 2}(t-\tau)^{-1 / 2} .
\end{aligned}
$$

On the other hand,

$$
\hat{\hat{v}}=\int_{\tau}^{t} \int_{-\infty}^{\infty} G_{2}(x, t-s ; y)(u v)_{x}(y, s) d y d s=O(1) \tau^{-1 / 2}\left[K_{C}^{+}(x,+1)+K_{C}^{-}(x,+1)\right]
$$

by the same calculations used in the proof of Proposition 3.2, so that

$$
\|\hat{\hat{v}}\|_{L^{1}}=O(1) \tau^{-1 / 2} \text {. }
$$

Alternatively, (5.30) follows from the cruder bound (cf. Zu.1)

$$
\|\hat{v}\|_{L^{1}} \leqq \int_{\tau}^{t}\left\|(u v)_{x}\right\|_{L^{1}} d s
$$

and the sharpened derivative bound $\left\|(u v)_{X}\right\|_{L^{1}}=O(1) t^{-3 / 2}$ given in the next section.

Combining (5.25-5.26) and (5.30), we have

$$
\left\|v(x, t)-\left[\rho_{\tau}^{+} K^{+}(x, t)+\rho_{\tau}^{-} K^{-}(x, t)\right]\right\|_{L^{1}}=O(1)\left[\tau^{1 / 2}(t-\tau)^{-1 / 2}+(\tau+1)^{-1 / 2}\right] .
$$


Letting $t \rightarrow \infty$ in (5.31), we find that

$$
\begin{aligned}
\left|\rho_{\bar{\tau}}^{-}-\rho_{\tau}^{-}\right|+\left.\right|_{\bar{\tau}} ^{+}-\rho_{\tau}^{+} \mid= & \lim _{t \rightarrow \infty} \|\left[\rho_{\bar{\tau}}^{+} K^{+}(x, t)+\rho_{\bar{\tau}}^{-} K^{-}(x, t)\right] \\
& -\left[\rho_{\tau}^{+} K^{+}(x, t)+\rho_{\tau}^{-} K^{-}(x, t)\right] \|_{L^{1}} \\
= & O(1) \tau^{-1 / 2},
\end{aligned}
$$

for any $\bar{\tau} \geqq \tau$. Thus, the sequences $\left\{\rho_{\tau}^{ \pm}\right\}$are Cauchy, and approach limits $\rho_{\infty}^{ \pm}$at the rate $\tau^{1 / 2}$

Combining with (5.31), and setting $\tau=t^{1 / 2}$, we have

$$
\begin{aligned}
\left\|v(x, t)-\left[\rho_{\infty}^{+} K^{+}(x, t)+\rho_{\infty}^{-} K^{-}(x, t)\right]\right\|_{L^{1}} & =O(1)\left[\tau^{1 / 2}(t-\tau)^{-1 / 2}+(\tau)^{-1 / 2}\right] \\
& =O(1) t^{-1 / 4},
\end{aligned}
$$

as claimed.

Remark. 1. Similar calculations give $L^{\infty}$ bounds. Equations (5.27-5.28) becomes $(t-\tau)^{-1}\|\cdot\|_{L^{1}}$, while (5.29) gives $\|\hat{\hat{v}}\|_{L^{\infty}}=O(1) \tau^{-1 / 2} t^{-1 / 2}$. Combining as before, we obtain the rate of $L^{\infty}$ convergence of $t^{-3 / 4}$. The rates of convergence in $L^{p}, 1<$ $p<\infty$, follow by interpolation between $L^{1}$ and $L^{\infty}$.

Remark. 2. A genuinely nonlinear term $v^{2}$ in the flux of $v$ is no obstacle to the above analysis. We obtain convergence at the same rates to a pair of "nonlinear heat kernels" (scale invariant solutions of Burgers eqution), as in [Zu.1].

\section{Bounds on Higher Derivatives}

The arguments given in the previous sections can be applied almost word for word to give bounds on higher derivatives of the perturbation. The basic stability result from Chapter 4 becomes

Proposition 6.1. Let $C>1, t_{0}$ and $N$ be fixed, and let $\left|u^{0}(x)\right|,\left|v^{0}(x)\right| \leqq \delta e^{-x^{2} 4 t_{0}}$ and $\int_{-\infty}^{\infty} u^{0}(x) d x=0$. If $\delta$ is sufficiently small, then (4.1) has a global solution satisfying

$$
D^{n} u(x, t)=O(1) \delta\left[t^{-n / 2}\left(t+t_{0}\right)^{-1} W_{C}\left(x, t+t_{0}\right)+\left(t+t_{0}\right)^{-1 / 2} e^{-|x| / 2 C}\right]
$$

for $n<N$,

$$
D^{N} u(x, t)=O(1) \delta\left[t^{-N / 2}\left(t+t_{0}\right)^{-1} W_{C}\left(x, t+t_{0}\right)+\left(t+t_{0}\right)^{-1 / 2} e^{-|x| / 2 C}\right],
$$

and

$$
D^{n} v(x, t)=O(1) \delta t^{-n / 2}\left[K_{C}\left(x, t+t_{0}\right)+K_{C}^{+}\left(x, t+t_{0}\right)\right]
$$

for all $n \leqq N$, where $O(1)$ is a bound depending only on $C, t_{0}, N$.

The form of Proposition 6.1 illuminates the structure of our original argument somewhat. We see now that at level $N$, the argument gives sharp bounds on all $v$ derivatives, and all $u$ derivatives except for $D^{N} u$. We are able to "gain a derivative" in our argument because of strength of Proposition 3.2 gives sharp bounds on $v$ despite the non-optimal bound on $u$. 
Acknowledgement. Kevin Zumbrun thanks the Standford University Mathematics Department for its hospitality during the visit in which this paper was begun. Thanks also to Vicki Botos at Indiana university, for her efforts in typing the manuscript.

This work was supported in part by NSF grant DMS-9216725 and Army grant DAAL03-91G-0017 (Liu) and by NSF grant DMS-9107990 (Zumbrun).

\section{References}

[Che-X] Chern, I.L., Xin, Z.: Stability of inviscid complex Burgers shock. Unpublished calculation, (1989)

[Co-Fr] Courant, R., Friedrichs, K.O.: Supersonic Flows and Shock Waves, Berlin, Heidelberg, Newyork: Springer, 1948

[F] Freistuhler, H.: Dynamical stability and vanishing viscosity: A case study of a nonstrictly hyperbolic system of conservation laws. CPAM 45, 561-582 (1992)

[F-L] Freistuhler, H., Liu, T.P.: Nonlinear stability of overcompressive shock waves in a rotationally invariant system of viscous conservation laws. Commun. Math. Phys. 153, $147-158$ (1993)

[Gl] Glimm, J.: Solutions in the large for nonlinear hyperbolic systems of equations. Commun. Pure Appl. Math. 18, 697-715 (1965)

[G.1] Goodman, J.: Nonlinear asymptotic stability of viscous shock profiles for conservation laws. Arch. Rat. Mech. 95, 325-344 (1986)

[G.2. Goodman, J.: Remarks on the stability of viscous shock waves. In Viscous profiles and numerical methods for shock waves, ed: M. Shearer, Philadelphia, PA: SIAM, 1991, pp. 66-72

[H-Sm.] Hoff, D., Smoller, J.: Solutions in the large for certain nonlinear parabolic systems. Ann. Inst. Henri Poincare 23, 210-220 (1985)

[I-Ma-Pl] Isaacson, E., Marchesin, D., Plohr, B.: Transitional waves for conservation laws. SIAM J. Math. Nal. 21, 837-866 (1990)

[Lax] Lax, P.: Hyperbolic systems of conservation laws II. Commun. Pure Appl. Math. 10, 537-566 (1957)

[L.1] Liu, T.-P.: Nonlinear stability of shock waves for viscous conservation laws. Mem. Am. Math. Soc. 56, 328, 1-108 (1985)

[L.2] Liu, T.-P.: Admissible solutions of hyperbolic conservation laws, Memoirs, Am. Math. Soc. No, 240 (1981)

[L.3] Liu, T.-P.: On the viscosity criterion for hyperbolic conxervation laws. In Viscous profiles and numerical methods for shock waves, ed.: Shearer, M., Philadelphia: SIAM, 1991, pp. 105-114

[L.4] Liu, T.-P.: Pointwise convergence to viscous shock and diffusion waves of conservation laws. To appear.

[L-Xi] Liu, T.-P., Xin, Z.: Stability of viscous shock waves associated a system of nonstrictly hyperbolic conservation laws. CPAM 45, 361-388 (1992)

[Mat-Ni] Matsumura, A., Nishihara, K.: On the stability of travelling wave solutions of a one dimensional model system for compressible viscous gas. Japan J. Appl. Math 2, 17-25 (1985)

[Sat] Sattinger, D.: On the stability of waves of nonlinear parabolic systems. Adv. Math. 22, 312-355 (1976)

[Sch-Sh] Schaeffer, D., Shearer, M.: The classification of $2 \times 2$ systems of no-strictly hyperbolic conservation laws, with application to oil recovery. J. Comm. Pure Appl. math. 40, $141-178$ (1987)

[Sz-X] Szepessy, A., Xin, Z.P.: Nonlinear stability of viscous shock waves, Report TRITANA-9201, Royal Institute of Technology, S-100 44 Stockholm (1992)

[Zu.1] Zumbrun, K.: Formation of diffusion waves in a scalar conservation law with convection. To appear, AMS Proc. (1993)

[Zu.2] Stability of general inviscid shock. Unpublished calculation (1990)

[Zu-Pl-Ma] Zumbrun, K., Plohr, B., Marchesin, D.: Scattering behavior of transitional shock waves. Mat. Contemp. 3, 191-209 (1992) 